SMOKING CESSATION

\title{
Bupropion and the risk of sudden death: a self- controlled case-series analysis using The Health Improvement Network
}

\author{
R Hubbard, S Lewis, J West, C Smith, C Godfrey, L Smeeth, P Farrington, J Britton

See end of article for authors' affiliations

Correspondence to Dr R Hubbard, Clinical Sciences Building City Hospital, Hucknall Road, Nottingham NG5 1PB, UK; Richard.Hubbard@ Nottingham.ac.uk

Received 1 February 2005 Accepted 30 June 2005 Published Online First 29 July 2005
Background: Bupropion is an effective smoking cessation therapy but its use in the UK has been limited by concerns that it may increase the risk of sudden death.

Methods: Data for all patients prescribed bupropion within The Health Improvement Network la computerised general practice database) were extracted and the self-controlled case-series method was used to estimate the relative incidence of death during the first 28 days of treatment. The incidence of seizures, a recognised adverse effect of bupropion, was also investigated during this period.

Results: A total of 9329 individuals had been prescribed bupropion (mean age 44 years, $48 \%$ male). The total person-time after the first prescription for bupropion was 17586 years, and during this time 121 people died. Two people died within the first 28 days of treatment, which was less than expected in comparison with the remaining observation period by an incidence ratio of $0.50195 \%$ confidence interval (CI) 0.12 to 2.05). Twenty eight people were recorded as having a total of 45 seizures ( 23 before starting bupropion, two in the first 28 days of treatment, and 20 at a later point). The relative incidence of seizures during the first 28 days of treatment was 3.62 (95\% Cl 0.87 to 15.09), equivalent to one additional seizure per 6219 first time bupropion users.

Conclusions: Bupropion use is probably associated with an increased risk of seizures, but no evidence was found to suggest that the drug is associated with an increased risk of sudden death.
C garette smoking is the most important preventable cause of morbidity and premature mortality in the UK, and smoking cessation interventions-including nicotine replacement therapy or bupropion-are among the most cost effective healthcare interventions available. ${ }^{12}$ Bupropion was launched in the UK in June 2000 and there was a rapid uptake in the use of this drug. In February 2001, however, a newspaper report suggested that bupropion caused an increased risk of sudden death, and this was followed by a series of other anecdotal reports in the tabloid press. ${ }^{3}$ The use of bupropion in the UK has since been very low.

Smoking cessation is the only intervention which has been shown to reduce the loss of lung function in people with chronic obstructive pulmonary disease. ${ }^{4}$ Given the limited range of treatments available for smoking cessation, we believe that more robust evidence on the association between bupropion and sudden death is required before this treatment option is discarded by health professionals in the UK. We have therefore analysed data from a new general practice database (The Health Improvement Network $\left.(\text { THIN })^{5}\right)$ and, to prevent confounding resulting from interperson comparisons, we analysed the data using the selfcontrolled case-series method which compares the incidence of an outcome during a "high risk period" after drug exposure to other time periods within person..$^{6-8}$

\section{METHODS}

THIN contains computerised longitudinal primary care data collected in the process of routine clinical care. ${ }^{5}$ The data are downloaded from general practices which use the Vision software package supplied In Practice Systems. The information contained within THIN includes information on all prescriptions supplied by the general practitioner, all diagnoses made by or reported to the general practitioner, as well as other information relevant to primary care such as smoking habit and body mass index. The data for the present study were extracted as part of a wider study of the safety of smoking cessation interventions including nicotine replacement therapy and include data up to November 2003. At the time of the data extraction, THIN included data for more than 3.1 million patients registered at 221 practices.

For our main statistical analyses we used the case-series method, ${ }^{67}$ which is a self-controlled method in which the incidence of an outcome during a "high risk" exposure time is compared with the incidence during the remaining "control" time within person. The control time may include time both before and after the high risk time. Since the caseseries method is based on exposed people with the outcome of interest, it is an efficient design which provides the same amount of statistical power as an analysis of an equivalent whole cohort and slightly more statistical power than an equivalent nested case-control study. ${ }^{6}$ The main advantage of the case-series method over a traditional cohort method for the present study is that it removes the problem of confounding caused by factors that vary between people, such as the extent of coronary artery disease. This means that the case-series approach allows for the fact that smokers prescribed bupropion may represent a select group who are more likely to have smoking related illnesses.

Initially we identified all people with at least one prescription for bupropion during their THIN data record, and this was the baseline cohort used for all subsequent analyses. Within this cohort for each person we identified the start of the computerised record, the date of all prescriptions for bupropion, the date of death where present, and the date of the last data collection. Using these data we calculated the crude death rate for the follow up period after the first prescription for bupropion and used Poisson regression (STATA version 7) to compare the crude death rate during the 28 days after the first prescription for bupropion with 
that in the remaining person-time. We chose 28 days as this is the duration of bupropion treatment currently recommended. ${ }^{1}$ For our case-series analyses we defined the high risk period as a period of 28 days after the first prescription for bupropion and the control time as the time between the end of the high risk period and the date of last data collection in the cohort. Since people had to be alive to receive a prescription for bupropion, we did not include any persontime before the first prescription for bupropion in this analysis and, since we were interested in the theoretical risk of dying at any point during the study period, each person who died was given the same end date of 11 November 2003, the date of last data collection. We compared the risk of dying during the high risk period with that during the control period using the case-series method (GLIM version 4.9), adjusting for the effects of age in 2 year age bands. Although we chose a 28 day "high risk period" to reflect current treatment recommendations, ${ }^{1}$ we also repeated our analyses using a 63 day high risk period, which corresponds to the maximum recommended duration of bupropion treatment, in order to check the robustness of our findings.

Since bupropion (in common with other antidepressants) is a recognised cause of seizures, ${ }^{9}$ we repeated our analyses using seizure as an additional outcome. For these analyses we used all of the follow up time including that before the use of bupropion, since people can have multiple seizures.

The study protocol was approved by the South East multicentre research ethics committee.

\section{RESULTS}

The study cohort included 9329 individuals who had at least one prescription for bupropion during their THIN record. The mean age of the cohort at first prescription was 44 years and $4501(48 \%)$ were male. The plot of the frequency of bupropion prescriptions over time shows that the crude number of daily prescriptions recorded in the dataset increased rapidly during the first 8 months after the drug launch but declined sharply after the adverse publicity (fig 1).

The total follow up period after the first bupropion prescription was 17586 person-years (mean (SD) 1.9 (0.9) years), and during this time there were 121 deaths. This is equivalent to a crude death rate in our cohort of 70 per 10000 person-years or approximately 1.2 deaths per week in the cohort. During the 28 day high risk period after starting bupropion two people died; the Poisson regression comparison of the crude death rate during this period with that during the remaining person-time gave an incidence ratio of 0.40 (95\% confidence interval (CI) 0.10 to 1.60 ). The caseseries age adjusted estimate of the relative incidence of death

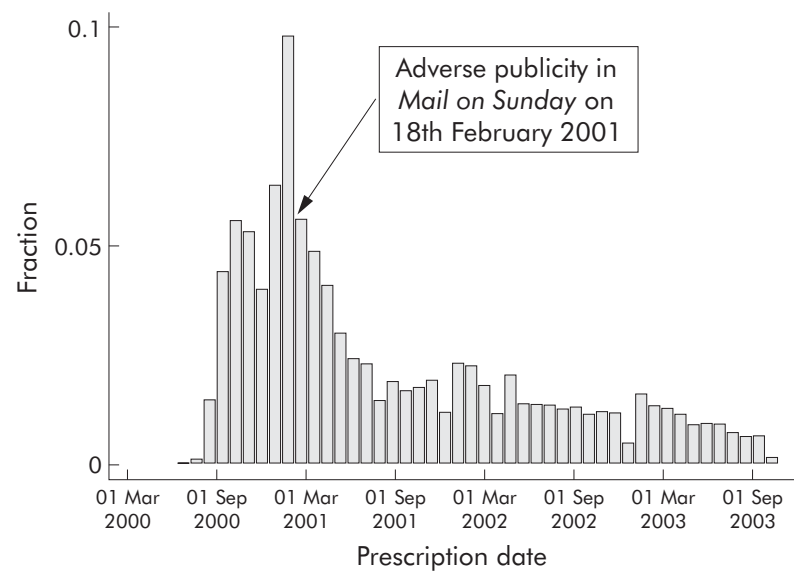

Figure 1 Distribution of first prescriptions for bupropion over time. during the high risk period was similar at 0.50 (95\% CI 0.12 to 2.05 ). The equivalent results for the 63 day high risk period were five deaths and a case-series estimate of relative incidence of 0.47 (95\% CI 0.18 to 1.19 ).

Using the whole of the THIN record for people both before and after the first prescription for bupropion, the total follow up time was 82935 years (mean (SD) 8.9 (4.2) years). During this time 28 people were recorded as having at least one seizure, and 45 diagnoses of fits were recorded in total. The overall crude seizure rate in our cohort was thus 5 per 10000 person-years, equivalent to one seizure in the cohort every 10 weeks. Twenty three seizures were recorded before the first prescription, two in the 28 days after the prescription, and 20 at a later time. The two seizures recorded during the first 28 days of treatment occurred on days 5 and 6 in one person who had no previous history of epilepsy. The Poisson regression comparison of the crude seizure rates during this period compared with the remaining person-time gave an incidence ratio of 5.35 (95\% CI 1.23 to 22.1) Our case-series age adjusted estimate of the relative incidence of seizures following bupropion was slightly lower at 3.62 (95\% CI 0.87 to 15.09). The equivalent results for the 63 day high risk period were three seizures and a case-series relative incidence of 2.38 (95\% CI 0.72 to 7.93 )

\section{DISCUSSION}

This is the largest safety study of first time users of bupropion reported to date. Our findings confirm that, although not statistically significant for our case-series analysis, there is probably an increased risk of seizures associated with bupropion use. However, we found no evidence of an increased risk of sudden death and, if anything, the mortality rate while using bupropion was lower than expected.

The main advantages of using general practice datasets such as THIN for post-marketing safety studies is that they produce statistically powerful datasets with detailed and accurate prescribing data. ${ }^{5}{ }^{10}$ The main potential disadvantage of these datasets is the presence of bias arising from the lack of data on other important variables such as the presence and severity of ischaemic heart disease. It seems likely that smokers who are prescribed bupropion will differ from those who are not and, in particular, are more likely to have progressive smoking related illnesses such as COPD or cardiovascular disease which may have precipitated the consultation and the resulting prescription. This means that it is likely that classical epidemiological methods, which use smokers not exposed to bupropion as a comparator group, will produce an overestimate of the adverse events attributed to the use of bupropion. In these circumstances the use of self-controlled methods, such as the case-series method, will remove this bias and produce more valid estimates of the relative increase in adverse events directly attributable to the drug. We have previously reported a similar phenomenon in a study of the impact of antidepressants on hip fracture. ${ }^{8}$ In the event, for this study the estimates of relative incidence for mortality in the first 28 days after starting bupropion treatment compared with a later point in time were very similar whichever method was used. For seizures, however, the case-series estimates were lower than that seen for the crude Poisson analysis. The likely reason for this difference is the presence of confounding by age and co-morbid illness, which is allowed for in the case-series analysis but not in the Poisson analysis.

The validity of the outcome data is another important consideration for our study, particularly the completeness and accuracy of the recording of deaths and seizures. A recent study of the recording of death in THIN has shown that the death rate in the dataset is 10.3 per 1000 person-years, which is very similar to the figure of 10.2 from the Office of National 
Statistics and suggests that the validity of these data is high. ${ }^{5}$ There have been no specific studies of the validity of seizure diagnoses in THIN, so it is possible that these data are not as complete as those for death registration. However, given the concern in the media about the adverse effects of bupropion, it seems likely that the ascertainment of seizures during the period of treatment with bupropion will tend to be higher than that during other times so, if any variations in ascertainment exist, these will, if anything, lead to an overinflation of the adverse impact of bupropion on seizure rate.

Bupropion was first used for the treatment of depression in the late 1980s and at this time was only available in an immediate release form. ${ }^{11}$ In an open label trial of 3341 patients using up to $450 \mathrm{mg} /$ day of this formulation, the risk of seizure during the first 8 weeks of treatment was $0.36 \% .{ }^{12}$ In 1996 a sustained release formulation was introduced and, in a similar open label trial involving 3100 patients and a maximum daily dose of $300 \mathrm{mg}$, three people $(0.1 \%)$ had a seizure. ${ }^{9}$ This increased risk of seizure is similar to that found for other commonly used antidepressants. ${ }^{13}$ In the UK for smoking cessation the sustained release preparation is used at a maximum dose of $300 \mathrm{mg}$ and the suggested risk of seizure is $0.1 \%{ }^{1}$ Bupropion avoidance or a maximum daily dose of $150 \mathrm{mg}$ is recommended if there is a risk of seizures, so it is likely that some selection is present in our cohort with people considered to be at high risk of seizures excluded. This exclusion was not complete, however, since 12 people had a total of 23 seizure diagnoses recorded before the first bupropion prescription. In our dataset there were two seizures during the first 28 days after starting treatment with bupropion and only 0.5 seizures were expected. This is equivalent to an additional one seizure per 6219 first time bupropion users, suggesting that bupropion has a better safety profile in relation to seizure than previously reported.

There are few data available on the association between bupropion and mortality, but a systematic review of the limited data available from clinical trials involving bupropion for smoking cessation has found no evidence to support an increase in mortality. ${ }^{14}$ In a questionnaire survey by the UK Drug Safety Research Unit, general practitioners were asked to provide information on bupropion users. Fourteen deaths were reported among 11735 replies (response rate of 48\%) during the first 12 weeks after starting bupropion treatment. ${ }^{15}$ Using data for American smokers as a comparator population, this was equivalent to a standardised mortality ratio of 0.77 (95\% CI 0.42 to 1.28). Despite the differences in study design and the limitations of the control population, these results are similar to our own in demonstrating no increase in mortality in relation to the use of bupropion. In fact, both studies have shown a non-significant decrease in mortality in the period after starting bupropion treatment. The reasons for this are not clear, but may reflect a tendency for general practitioners to prescribe bupropion during times when patients are generally well and not to initiate treatment during periods of acute illness.

This study describes the experience of using bupropion in primary care in the UK. The results show that the drug is probably associated with a small increase in seizure rate, which is similar to that seen with most other antidepressants when used to treat depression. In a cohort of 9329 first time users of bupropion we found no evidence to suggest that the drug is associated with an increased risk of sudden death, and our findings suggest that a hesitancy to use the drug on these grounds is unfounded.

\section{ACKNOWLEDGEMENTS}

The authors thank Alan Dean, Hassy Dattani, Alison Bourke, and Louise Pinder from the Epidemiology and Pharmacology Information Core (EPIC) for their expert advice in using THIN.

\section{Authors' affiliations}

R Hubbard, J West, J Britton, Division of Epidemiology and Public

Health, University of Nottingham, Nottingham, UK

S Lewis, C Smith, Division of Respiratory Medicine, University of

Nottingham, Nottingham, UK

C Godfrey, Centre for Health Economics, University of York, York, UK

L Smeeth, Department of Epidemiology and Population Health, London

School of Hygiene and Tropical Medicine, University of London, London, UK

P Farrington, Department of Statistics, Open University, Milton Keynes, UK

This project was funded by the British Lung Foundation and the Wellcome Trust.

Competing interests: RH is a member of The Health Improvement Network (THIN) advisory group. JB has received an honorarium from a third party organisation but originating from GlaxoSmithKline (the producers of bupropion) for speaking at an invited conference on smoking cessation management guidelines in 2002, and also received consulting fees from the manufacturers of nicotine replacement therapy and other novel smoking cessation therapies.

$\mathrm{RH}, \mathrm{JB}$ and $\mathrm{LS}$ were responsible for the main study hypothesis and $\mathrm{RH}$, $\mathrm{SL}, \mathrm{CS}, \mathrm{CG}, \mathrm{LS}, \mathrm{PF}$ and JB were responsible for the detailed study design and were applicants on the British Lung Foundation grant. The analysis was performed by RH, JW, SL, PF and CS. All authors contributed to the writing of the paper and all authors have had access to all of the data in the study.

Work attributed to Division of Epidemiology and Public Health, Nottingham University.

\section{REFERENCES}

1 West R, McNeill A, Raw M. Smoking cessation guidelines for health professionals: an update. Thorax 2000;55:987-99.

2 Tobacco Advisory Group of the Royal College of Physicians. Nicotine addiction in Britain. London: Royal College of Physicians of London, 2000.

3 Ellis R. Smokers die after taking Zyban cure. Mail on Sunday 18 February 2001.

4 Anthonisen NR, Connett JE, Kiley JP, et al. Effects of smoking intervention and the use of an inhaled anticholinergic bronchodilator on the rate of decline of FEV. . JAMA 1994;272:1497-505.

5 Bourke A, Dattani H, Robinson M. Feasibility study and methodology to create a quality-evaluated database of primary care data. Inform Prim Care 2004:12:171-7.

6 Farrington CP, Nash J, Miller E. Case series analysis of adverse reactions to vaccines: a comparative evaluation. Am J Epidemiol 1996;143:1 165-73.

7 Farrington P, Pugh S, Colville A, et al. A new method for active surveillance of adverse events from diptheria/tetanus/pertusis and measles/mumps/rubella vaccines. Lancet 1995;345:567-9.

8 Hubbard R, Farrington $P$, Smith $C$, et al. Exposure to tricyclic and selective serotonin reuptake inhibitor antidepressants and the risk of hip fracture. Am J Epidemiol 2003;158:77-84.

9 Dunner DL, Zisook S, Billow AA, et al. A prospective safety surveillance study for bupropion sustained release in the treatment of depression. J Clin Psychiatry 1998;59:366-73.

10 Walley T, Mantgani A. The UK General Practice Research Database. Lancet 1997;350:1097-9.

11 Settle EC Jr. Bupropion sustained release: side effect profile. J Clin Psychiatry 1998;59(Suppl 4):32-6.

12 Johnston JA, Lineberry CG, Ascher JA, et al. A 102-center prospective study of seizure in association with bupropion. J Clin Psychiatry 1991;52:450-6.

13 Pisani F, Oteri G, Costa C, et al. Effects of psychotropic drugs on seizure threshold. Drug Safety 2002;25:91-1 10.

14 Hughes J, Stead L, Lancaster T. Antidepressants for smoking cessation. Cochrane Database Syst Rev 2004;(4):CD000031.

15 Boshier A, Wilton LV, Shakir SA. Evaluation of the safety of bupropion (Zyban) for smoking cessation from experience gained in general practice use in England in 2000. Eur J Clin Pharmacol 2003;59:767-73. 